\title{
Auto e hetero-avaliação no diagnóstico de necessidades de treinamento
}

\author{
Mônica Lemes Magalhães \\ Faculdade Garcia Silveira \\ Jairo Eduardo Borges-Andrade \\ Universidade de Brasília
}

\begin{abstract}
Resumo
O presente estudo teve como objetivos: desenvolver uma metodologia de avaliação de necessidades de treinamento que incluísse atitudes; estudar a relação entre auto e hetero-avaliação, buscando avaliar conhecimentos, habilidades e atitudes; e avaliar a relação entre a auto-avaliação e algumas variáveis preditoras (tempo de trabalho na organização e na função, emprego anterior, gênero, estado civil, escolaridade e idade). Foram desenvolvidos questionários, de acordo com o método de análise de papel ocupacional. Esses questionários foram aplicados em 898 bancários e seus supervisores imediatos. Os resultados sugeriram que a metodologia parece ser adequada para analisar necessidades de treinamento. Mostraram também que existe relação significativa entre auto e hetero-avaliação de necessidades de treinamento, embora supervisores tendam a apontar maiores necessidades de treinamento, em seus subordinados, do que as auto-avaliações destes mesmos.
\end{abstract}

Palavras-chave: Treinamento, Necessidades de treinamento, Avaliação de necessidades de treinamento, Auto-avaliação, Hetero-avaliação.

\begin{abstract}
Self and hetero assessment in training needs diagnostic

The objectives of the present study were: to develop a training needs assessment methodology that included attitudes; to study the relationship between self and hetero assessment, by assessing knowledge, skills and attitudes; and to evaluate the relationship between self-assessment and some predicting variables (work experience at the organizational and occupational levels, prior job experience, gender, marital status, schooling and age). Questionnaires were developed, following the method of occupational role analysis. These questionnaires were applied in 898 bank employees and their immediate supervisors. Results have suggested that the methodology seems to be appropriate for analyzing training needs. They have also shown that there is a significant relationship between self and hetero assessments of training needs. However, supervisors tend to point higher training needs, in their subordinates, than the self-assessments made by the latter.
\end{abstract}

Key words: Training, Training needs, Training needs assessment, Self-assessment, Hetero-assessment.

\section{Treinamento e tecnologia de treinamento}

$\mathrm{A}$ velocidade das mudanças tecnológicas, o aumento da diversidade nos locais de trabalho e a acentutos do mundo contemporâneo que justificam os elevados investimentos em treinamento e estão presentes na literatura científica que o investiga. De acordo com Schneider (1999), estas são algumas razões pelas quais o treinamento se tornou uma questão crucial para as organizações.

A palavra treinamento tem muitos significados. Alguns especialistas em administração de pessoal consideram o treinamento como um meio para desenvolver a força de trabalho dentro dos cargos particulares (Yoder, 1956). Outros interpretam mais amplamente, considerando o treinamento para 
um adequado desempenho no cargo e estendendo o conceito para um nivelamento intelectual através da educação geral (Waite, 1952). Chiavenato (1992) define como sendo um processo educacional de curto prazo aplicado de maneira sistemática e organizada, através do qual pessoas aprendem conhecimentos, habilidades e atitudes em função de objetivos definidos. Wexley (1984) refere-se a treinamento como um esforço planejado de uma organização para facilitar a aprendizagem de seus integrantes. Há, portanto, várias maneiras de definir treinamento. A que parece ser a mais objetiva e que engloba de certa maneira as outras definições é a de Goldstein (1991), que propõe ser treinamento uma aquisição sistemática de atitudes, conceitos, conhecimentos, regras e habilidades que resultam no melhor desempenho no trabalho.

As definições dadas identificam o treinamento como sendo uma forma de educação especializada, uma vez que seu propósito é preparar o indivíduo para o desempenho eficiente de uma determinada tarefa que lhe é atribuída. Nesta linha de pensamento, o treinamento é uma função organizacional e inclui um "somatório de atividades que vão desde a aquisição de habilidade motriz até o desenvolvimento de um conhecimento técnico complexo, à assimilação de novas atitudes administrativas e à evolução de comportamento em função de problemas sociais complexos" (McGehee \& Tahyer, 1961, p. 7). Carvalho (1999), ao analisar os conceitos atuais de treinamento, reconhece que esta função organizacional passou por um processo de sofisticação. Contudo, seu processo básico continuaria o mesmo: é preciso ter clareza do que vai ser ensinado, diagnosticar as necessidades dos que irão aprender, oferecer situações de ensino e, finalmente, verificar os resultados.

O campo de treinamento de recursos humanos é de maneira geral imediatamente associado ao planejamento sistemático de eventos instrucionais. Tal processo se baseia nos subsídios provenientes da análise dos requisitos do trabalho e das capacidades do treinando, assim como nos princípios da tecnologia instrucional, com o objetivo de garantir as condições ótimas para a aquisição dos conhecimentos, habilidades e/ou atitudes necessárias ao exercício de um dado papel ocupacional.

Segundo Borges-Andrade (1986), uma tecnologia de treinamento poderia ser definida como o conjunto de princípios e prescrições que se aplicariam a essa função. Esse conjunto deveria ser formado por partes ou elementos coordenados entre si e que funcionassem em uma estrutura organizada, que pudesse oferecer alternativas eficazes, mesmo quando novos problemas se apresentassem. O enfoque instrucional, geralmente adotado como base dessa tecnologia, pode ser definido como o modo como os profissionais de educação ou treinamento conhecem, aprendem e predizem as coisas concernentes ao ensino e à aprendizagem, bem como à relação entre as mudanças de desempenho que se deseja de um determinado indivíduo e o que se faz com o propósito de obter essas mudanças. Este enfoque se baseia nos conhecimentos advindos da psicologia da aprendizagem e teoria de sistemas.

Nasce daí um ramo relativamente novo da ciência, denominado psicologia instrucional. O modo como essa ciência é usada, para resolver problemas em treinamento, é denominado tecnologia instrucional. Essa tecnologia prevê que o processo de treinamento é composto de quatro etapas, que são a avaliação de necessidades, o planejamento, a execução e a avaliação do treinamento. O foco do presente texto é na primeira destas etapas.

\section{Avaliação de necessidades de treinamento}

A avaliação de necessidades de treinamento subsidia o planejamento. Uma "necessidade de treinamento pode ser descrita como a existência, em qualquer tempo, de uma condição real que difere de uma condição desejada nos aspectos humanos ou pessoais, do desempenho de uma organização, ou mais especificamente, quando uma mudança nos conhecimentos, habilidades ou atitudes humanas atuais pode levar ao desempenho desejado" (Morrison, 1977, citado por Nogueira, 1982, p. 06).

McGehee e Thayer (1961) descreveram três componentes básicos da avaliação de necessidades: análise organizacional, análise de tarefas e análise pessoal. Tais componentes receberam, anos depois, uma nova conceituação feita por Goldstein (1991), que se mantém atual e é usada como quadro de referências útil para a compreensão do processo de avaliação de necessidades (Kozlowiski, Brown, Weissbeim, Cammon-Bowers \& Salas, 2000). Por essas razões, essa conceituação será apresentada nos quatro próximos parágrafos.

O primeiro componente refere-se à análise organizacional, que focaliza o papel do treinamento em um nível de análise macro. $\mathrm{O}$ objetivo deste componente é conhecer a realidade da organização, o que envolve o exame do sistema organizacional como um todo. É aí incluído o estudo de objetivos atuais e futuros, clima, cultura, estrutura, fluxo, situação econômico-financeira, tecnologia em uso, além da interação da instituição com o meio externo, o que abrange mercado de trabalho, impacto de produtos e serviços, concorrência, fornecedores e conjuntura sócio-econômica e política. A análise organizacional é um componente muito importante dentro da avaliação de necessidades, pois o sistema organizacional pode afetar o treinando antes e depois do treinamento.

Pretende-se, neste nível, analisar as causas e propor possíveis soluções para reais problemas levantados ou, ain- 
da, detectar situações que indiquem um potencial para crescimento e desenvolvimento. Tal análise permite a determinação de quais pontos podem ser resolvidos através de treinamento ou através de outras estratégias. No nível organizacional, geralmente ocorre muita dificuldade não só na identificação das necessidades de treinamento como também na definição dos objetivos do treinamento. Mas, como um sistema aberto, o sistema de treinamento não deve se manter isolado do contexto organizacional que o envolve e dos objetivos empresariais que lhe definem a direção. Assim, os objetivos do treinamento precisam estar ligados intimamente às necessidades da organização.

Em um nível de análise mais "micro", a avaliação de necessidades é realizada através do exame das tarefas relativas aos diversos papéis ocupacionais e às capacidades do indivíduo ao exercê-los (Paula, 1992). A análise de tarefas resulta na descrição de uma seqüência de atividades ou operações desempenhadas no trabalho e nas descrições das condições em que este trabalho é desempenhado. Em princípio, o treinamento surge como uma possível solução para uma discrepância de desempenho. Mager e Pipe (1977, citado por Paula, 1992) referem-se à discrepância como sendo a diferença entre uma condição atual e outra que se deseja alcançar. O treinamento deve ser considerado como uma solução legítima somente quando o diagnóstico da causa da discrepância estiver relacionado a uma lacuna de conhecimentos, habilidades e/ou atitudes necessários ao desempenho das atividades em questão. Como será demonstrado mais adiante, é neste nível de análise que se encontra a maior parte da produção científica da área, fato que já foi também confirmado numa análise da produção nacional feita por Borges-Andrade e Abbad-Oc (1996).

O último componente para avaliar necessidades procura verificar quais empregados precisam de treinamento e exatamente que treinamento é solicitado. Quando este estágio é alcançado, foi realizada uma análise organizacional que permitiu entender em que o sistema de treinamento pode apoiar a organização e quais facilitadores e inibidores existem, e uma análise de tarefas já determinou quais delas são importantes e não são desempenhadas. A análise pessoal faz duas perguntas: quem dentro da organização precisa de treinamento e que tipo de instrução precisa? Esta análise pode então ser direcionada para a realização de treinamentos específicos para determinadas pessoas.

Após a avaliação de necessidades, deve-se analisar a natureza do desempenho envolvido em cada necessidade, bem como as condições sob as quais ele deve aparecer. Este processo pode alimentar-se, para a execução, de dados fornecidos de análises organizacionais, de descrições de cargos e de avaliação de desempenho. Além disso, de acordo com Borges-Andrade (1986), ele efetivamente aponta para o que é relevante treinar, focaliza a atenção em desempenhos e torna as pessoas (da organização) que dele participam responsáveis por ele e envolvidas politicamente com seus resultados. A avaliação de necessidades é um momento para se analisar, a priori, o que deve ser treinado, para quem o treinamento serve e por que se investe nele. A organização pode utilizá-la como instrumento para discutir seus segmentos, seus papéis e o que deve ser mudado na mesma. Este autor define necessidades como as discrepâncias existentes entre os desempenhos esperados e reais. Com base nessa conceituação, o processo de avaliação de necessidades deveria incluir, inicialmente, a definição dos conhecimentos, habilidades e atitudes esperadas dos empregados e, em seguida, a mensuração dos níveis em que o indivíduo domina esses conhecimentos, habilidades e atitudes e da importância de cada uma delas. Esta insistência em incluir as categorias "conhecimento", "habilidades" e "atitudes", ou CHA, tem a sua origem na psicologia instrucional (Rothwell \& Kazanas, 1992) e pode ser identificada na própria definição já mencionada de treinamento, feita por Goldstein (1991). No entanto, este mesmo autor, cujos textos revelam uma forte influência da psicologia instrucional, nunca incluiu as atitudes em seu conhecido método de avaliação de necessidade de treinamento.

Entre os pesquisadores de treinamento, poucos discordam de que uma cuidadosa avaliação de necessidades da organização é de vital importância e deve ser feita antes de se desenvolver um programa de treinamento. Ostroff e Ford (1989), por exemplo, afirmam que a avaliação de necessidades de treinamento fornece informações sobre que treinamento é necessário, qual o conteúdo que o treinamento deve ter e quem, dentro da organização, precisa de treinamento em certos tipos de habilidades e conhecimentos. A literatura especializada tem ainda registrado que o insucesso de muitos programas de treinamento é devido principalmente à falta de uma avaliação adequada de necessidades. Byhan (1977) acentua que "sem um diagnóstico das necessidades das pessoas que estão sendo treinadas, é impossível conseguir-se um treinamento eficaz e, além disso, podemos estar treinando as pessoas erradas, sobre coisas erradas e em tempos errados" (p. 10).

Para fazer uma adequada avaliação de necessidades de treinamento, deve-se desenvolver instrumentos que estão de acordo com a realidade da organização, buscando colher informações que possam oferecer base para o processo de treinamento. No Brasil, Nogueira (1980) foi quem primeiro construiu e validou cientificamente um instrumento de identificação de necessidades de treinamento. Os resultados mostraram que a maioria dos itens possuía altos coeficientes de correlação com o respectivo fator e que os índices de precisão dos fatores eram elevados. 
Em 1983, Borges-Andrade e Lima desenvolveram um método de análise de papel ocupacional. Propuseram uma metodologia de avaliação de necessidades de treinamento que pode ser operacionalizada tendo-se como unidade de análise o papel ocupacional dos empregados na organização. Utilizaram como referencial teórico a abordagem de sistemas e os métodos, por ela influenciados, que surgiram no contexto da educação contemporânea. Criaram um instrumento que consistia de um questionário dirigido aos ocupantes de um dado papel ocupacional, que tinha a tarefa de descrever as habilidades necessárias para o desempenho do papel ocupacional em questão e posteriormente refinava as descrições feitas. Com base nesta metodologia, Menezes, Rivera e Borges-Andrade (1988) buscaram identificar prioridades de treinamento, através da opinião sobre a importância e domínio de uma lista de habilidades. Este trabalho foi dirigido para o desenvolvimento organizacional agrícola, especificamente para o desenvolvimento de recursos humanos no meio rural.

Borges-Andrade, Lima, Soares e Paula (1989), usando a mesma metodologia, estudaram necessidades de treinamento dos gerentes de pesquisa da EMBRAPA - Empresa Brasileira de Pesquisa Agropecuária - e o impacto dos seus treinamentos no desempenho de seus cargos. Concluíram que estas necessidades podem ser bastante diferenciadas, conforme o papel do gerente. Identificaram cada prioridade de treinamento, que foi a seguir considerada como variável dependente em modelos de regressão, em que características pessoais e organizacionais relativas aos gerentes eram os preditores hipotetizados. O teste destes modelos sugeriu que as necessidades dos gerentes, além de dependerem de seus papéis, parecem estar ligadas a outros fatores, sendo que um deles, a participação em cursos de gerência de pesquisa no passado, é relevante para se poder avaliar o impacto dos treinamentos já realizados. A metodologia de avaliação de necessidades utilizada ofereceu insumos para o planejamento de treinamento.

Existe uma variedade de fontes de dados e procedimentos para a avaliação das necessidades de treinamento. Morano (1973) argumentou que os empregados são conscientes de suas aptidões fracas e de seus desempenhos deficientes e, além do mais, estão na melhor posição para determinar suas próprias necessidades de treinamento. $\mathrm{O}$ uso de auto-avaliação para determinar necessidades de treinamento tem se tornado uma estratégia importante para as organizações.

Ford e Noe (1987) examinaram o impacto de três fatores sobre a auto-avaliação de necessidades de treinamento por empresários. Dois fatores, o nível hierárquico e a função, são características da posição do empresário na organização. O terceiro fator, atitude em relação à utilidade do treinamento, é uma característica individual. Como resultado desta pesquisa, os autores encontraram um efeito pequeno mas significativo para nível, função e atitudes sobre neces- sidades de treinamento relatadas. Empresários de nível baixo relataram maiores necessidades para habilidades administrativas que os empresários de nível médio. Empresários da área de fábrica e manipulação de materiais relataram maiores necessidades para treinamento de habilidades de qualidade que empresários com funções administrativas.

A partir dos estudos sobre auto-avaliação de necessidades de treinamento, alguns poucos pesquisadores (McEnery \& McEnery, 1987; Stanley \& Shockley-Zalabak, 1986) começaram a comparar auto-avaliações com hetero-avaliações, buscando verificar correlações ou diferenças entre esses tipos de avaliações. No Brasil, não foram encontrados relatos de investigações sobre estas questões.

Em 1986, Stanley e Shockley-Zalabak investigaram a percepção que o supervisor tinha da habilidade de comunicação de profissionais do sexo feminino, assumindo que são as habilidades de comunicação um componente crítico para a eficácia organizacional. Estes pesquisadores fizeram também a comparação entre a avaliação dos supervisores com uma variedade de habilidades de comunicação (entrevista, processo de decisão em grupo, apresentação oral, administração de conflitos, técnicas de liderança, relações interpessoais, tecnologia da comunicação - computador e telecomunicações, motivando pessoas, delegação, habilidade de negociação, diagnosticando problemas organizacionais) e a auto-avaliação das profissionais. Comparação similar foi feita entre a importância da avaliação do supervisor para treinamentos futuros com cada habilidade e a auto-avaliação das profissionais sobre suas necessidades. Para esta pesquisa foram sorteadas 122 profissionais do sexo feminino e 80 supervisores diretos, os quais haviam participado de um programa de desenvolvimento gerencial, de uma indústria privada e de agências do governo. Os resultados demonstraram as diferenças entre a percepção das profissionais e dos seus supervisores. De fato, os pesquisadores encontraram fraca relação entre a profissional e seu supervisor em ambos os termos pesquisados, habilidade de comunicação e necessidade de treinamento. Os autores sugerem que futuras pesquisas continuem explorando a comparação entre auto e hetero-avaliação.

McEnery e McEnery (1987) focalizaram também a utilidade de auto-avaliação comparada com a avaliação de necessidades de treinamento por supervisores. As auto-avaliações tenderam a ser mais indulgentes e evidenciaram ser menos tendenciosas que as avaliações feitas pelos supervisores dos subordinados. Auto-avaliações de necessidades resultaram em três fatores independentes, enquanto que as avaliações de necessidades pelos supervisores resultaram em apenas um fator geral. Esses resultados, casados com a evidência de que os supervisores projetaram suas próprias necessidades quando identificaram as necessidades dos subordinados, sugeriram que auto-avaliações de necessida- 
des podem ser um importante componente de um processo válido de avaliação de necessidades.

Em geral, as poucas pesquisas encontradas indicaram que há somente baixa para moderada correlação entre diferentes tipos de avaliação de desempenho e que a correlação entre auto-avaliação e avaliação pelo supervisor é particularmente fraca. Várias propostas foram oferecidas para explicar a falta de relação entre auto-avaliação e avaliação pelo supervisor. Foi sugerido que a inconsistência entre auto-avaliação e avaliação pelo supervisor pode ser esperada porque cada avaliador tem uma perspectiva única e focaliza diferentes facetas do desempenho no trabalho.

Enquanto a utilidade das auto-avaliações de necessidades de treinamento tem sido sugerida, há poucas pesquisas empíricas que façam comparações entre auto e hetero-avaliações. Estas comparações foram verificadas, nas poucas pesquisas realizadas, ora por correlações, ora por testes de diferenças entre médias. Estas duas formas de comparação são diferentes. Correlação é a medida do tamanho e direção da relação entre os dois tipos de avaliação. As correlações variam com respeito à sua força. $\mathrm{O}$ teste de diferenças entre médias, por outro lado, questiona se as médias das duas avaliações são diferentes. Observa-se, através da literatura, certa confusão no tratamento dos dados. Pesquisadores afirmam que as relações entre auto e hetero-avaliações não são significativas, ou que não existe relação entre elas, porém verifica-se diferenças no tratamento estatístico dos dados. Nas pesquisas revistas, observa-se que não foram feitas comparações entre auto e hetero-avaliação de necessidade de treinamento, utilizando-se simultaneamente de correlação e do teste de diferenças entre as médias das duas escalas.

Além disso, as pesquisas que tratam da avaliação de necessidades de treinamento, tanto nacionais como internacionais, têm enfocado habilidades e conhecimentos e não, trabalhado com atitudes. Até o presente momento, realizaram-se análises de tarefa que contemplam a relação entre função e habilidades/conhecimentos. Em nenhum momento se englobou a análise das atitudes, como parte dos requisitos para o bom desempenho em papéis organizacionais. Finalmente, neste processo não é somente relevante investigar necessidades, mas também é essencial verificar algumas possíveis variáveis que predizem a avaliação dessas necessidades feita pelos respondentes dos questionários.

\section{Objetivos da pesquisa}

Com base nas deficiências anteriormente apontadas, $o$ presente estudo teve como objetivos:

a) desenvolver uma metodologia de avaliação de necessidades que inclua atitudes;

b) estudar a relação entre auto e hetero-avaliação, buscando avaliar habilidades, conhecimentos e atitudes; e c) analisar a relação entre a auto-avaliação e algumas variáveis preditoras, que são: tempo de trabalho na organização, emprego anterior, tempo de trabalho no cargo atual, gênero, estado civil, escolaridade e idade.

Para efeito deste estudo, definiu-se necessidades de treinamento como "as discrepâncias existentes entre os desempenhos esperados e reais" (Borges-Andrade, 1986, p. 14), ou mais especificamente, "quando uma mudança nos conhecimentos, habilidades e atitudes humanas atuais pode levar ao desempenho desejado" (Morrison, 1977, citado por Nogueira, 1982, p. 6), na qual:

a) Conhecimento refere-se a um corpo organizado de informações de natureza técnica ou administrativa, o qual se aplicado faz com que o desempenho adequado do trabalho seja possível.

b) Habilidade refere-se à capacidade de desempenhar operações de trabalho com facilidade e precisão. Inclui comportamentos motores ou verbais que favorecem a realização das tarefas inerentes à função. As especificações das habilidades normalmente implicam um padrão de desempenho requerido para operações efetivas do trabalho.

c) Atitude é a predisposição do indivíduo que se manifesta verbalmente ou não, assumindo caráter de favorabilidade ou desfavorabilidade em relação a um objeto, pessoa ou fato, ou denota sentimentos do trabalhador a respeito do que ele faz ou sobre a organização em que trabalha ou alguma pessoa competente da mesma. Esta definição pode não ser compartilhada por muitos autores em Psicologia Social, mas é a que efetivamente tem sido usada, e há muito tempo, pelos teóricos da Psicologia Instrucional, bem como pelos profissionais que adotam este tipo de enfoque para planejar o ensino em situações educacionais e de treinamento para o trabalho (ver, por exemplo, Gagné \& Briggs, 1974; Goldstein 1991; Rothwell \& Kazanas, 1992).

A identificação dessas necessidades de treinamento, por sua vez, será realizada por auto-avaliação e por hetero-avaliação, sendo que:

a) Auto-avaliação é a avaliação feita do sujeito por ele mesmo.

b) Hetero-avaliação é a avaliação feita pelo supervisor do sujeito avaliado.

\section{Método}

Como parte do método da pesquisa, a seguir são descritos o universo e a amostra da mesma, depois os instrumentos e procedimentos de coleta de dados e, finalmente, os procedimentos usados para analisar estes dados.

\section{Universo e amostra}

A organização escolhida para a realização desta pesquisa foi a Caixa Econômica Federal (CEF) do Estado de 
Goiás. A escolha desta empresa ocorreu por dois motivos. Primeiro, pelo interesse da pesquisadora em avaliar necessidades de treinamento em uma instituição financeira. Segundo, pela necessidade da CEF em avaliar melhor as necessidades de treinamento de seus funcionários.

A $\mathrm{CEF}^{1}$ é uma instituição financeira, constituída sob a forma de Empresa Pública de Direito Privado, vinculada ao Ministério da Fazenda, possuindo patrimônio próprio e autonomia administrativa. Faz parte do sistema financeiro nacional, é órgão auxiliar da política de crédito do Governo Federal, sujeita-se às decisões e à disciplina normativa estabelecidas pelo Conselho Monetário Nacional e à fiscalização do Banco Central do Brasil.

A primeira Caixa Econômica foi criada em 22 de agosto de 1860 pelo Imperador D. Pedro II, na cidade do Rio de Janeiro. O Governo Imperial, oficializando as Caixas Econômicas, caracterizou-as como estabelecimentos de beneficência, dirigidos e administrados gratuitamente por diretores nomeados pelo Governo. A partir de 1864, o Governo Imperial autorizou o funcionamento das Caixas Econômicas nas províncias. Ainda no Império, as Caixas Econômicas ganharam autonomia e ampliaram as áreas de atividade.

As Caixas Econômicas Federais, até 1970, eram administradas regionalmente, sob a supervisão de um Conselho Superior sediado na cidade do Rio de Janeiro. Em $1^{0}$ de agosto de 1970, as Caixas Econômicas que atuavam nos 22 Estados foram unificadas em uma única instituição financeira (decreto-lei 579, de 12.08.69). A unificação foi concretizada a fim de proporcionar o fortalecimento interno da Empresa, a modernização de sua imagem e a expansão de suas operações tradicionais.

Em 1994, no Estado de Goiás, a CEF implantou o Programa de Qualidade Total, com a finalidade de melhorar a qualidade de suas atividades. Sua missão foi definida como: "Promover a melhoria contínua da qualidade de vida da sociedade, intermediando recursos e negócios financeiros de qualquer natureza, atuando, prioritariamente, no fomento ao desenvolvimento urbano e nos segmentos de habitação, saneamento e infra-estrutura e na administração de fundos, programas e serviços de caráter social". A CEF de Goiás abrange unidades na capital e demais cidades do Estado. Ao todo são 62 agências e 9 postos de atendimento bancário.

Foi selecionado para esta pesquisa o cargo de Caixa Executivo. Este cargo foi escolhido após entrevista com a Gerente do Departamento de Treinamento da CEF. A razão da escolha ocorreu por dois motivos: a conhecida insuficiência de treinamento para o cargo e por ser um dos cargos que não seria eliminado pela restruturação interna em andamento, não afetando com isso a realização da pesquisa.

Para a realização deste trabalho, utilizou-se como respondentes os ocupantes de cargos de Caixa Executivo e seus Supervisores. A CEF de Goiás tinha, na ocasião da pesquisa, no seu quadro de funcionários: 449 Caixas Executivos e 45 Supervisores, além de outros cargos que não são de interesse deste estudo.

\section{Instrumentos e procedimentos de coleta de dados}

Os questionários de auto-avaliação e hetero-avaliação, utilizados para a coleta de dados, foram inicialmente baseados nas descrições de cargo existentes na instituição pesquisada e em entrevistas de análise de tarefas realizadas para confirmar ou expandir estas descrições. O questionário de auto-avaliação para o cargo de Caixa Executivo, composto de 34 itens, foi distribuído da seguinte forma: 11 itens para conhecimento, 15 itens para habilidades e 8 itens para atitudes. O questionário de hetero-avaliação dos mesmos Caixas Executivos foi respondido pelos Supervisores imediatos. Um exemplar desse questionário se encontra no Apêndice A. O outro seguiu formato muito semelhante e não é apresentado aqui por falta de espaço, mas está apresentado em Magalhães (1996).

Em todos os questionários, após cada item de descrição do cargo havia duas escalas de cinco pontos cada uma, indo de 0 a 4 . Na primeira escala o respondente julgaria a importância destes itens (conhecimentos, habilidades e atitudes) para o bom desempenho da função. Na segunda escala, o respondente julgaria a quantidade de determinado conhecimento ou habilidade ou atitude já existente para desempenhar a função. Nesta segunda escala, houve diferenças nas definições de cada ponto, em função de especificidades determinadas pela natureza dos conceitos de atitudes, conhecimentos e habilidades. De qualquer modo, o intervalo de pontos também ia de 0 a 4 . Uma análise dos itens ou atividades listados sob cada uma das categorias - conhecimentos, habilidades e atitudes - revelará que processos psicológicos referentes às outras duas categorias sempre estarão envolvidos. Por exemplo, uma determinada atitude poderá depender de certos conhecimentos e habilidades relativos a alguma área de atuação do trabalhador; uma habilidade dependerá de prévia aquisição de conhecimentos específicos e da predisposição do trabalhador para usá-la (atitude). Essas inter-relações são reconhecidas aqui e têm sido fortemente discutidas na literatura sobre psicologia instrucional e sobre treinamento no trabalho.

No final de cada grupo de atividades descritas para conhecimentos, habilidades e atitudes, havia um espaço para que o respondente colocasse outras atividades não descritas e que ele próprio julgasse como sendo importantes para o bom desempenho da função. Este espaço foi colocado em todos os questionários, mas foi muito pouco usado pelos respondentes. 
As instruções contidas antes de cada grupo de atividades (conhecimentos, habilidades e atitudes) permitiam a dispensa de instruções orais adicionais. Os questionários de auto e hetero-avaliação continham também sua finalidade, que era a de "conhecer as necessidades de treinamento para o bom desempenho dos ocupantes dos cargos". O questionário de hetero-avaliação continha as mesmas escalas que os de auto-avaliação. Uma para avaliar a importância dos conhecimentos, habilidades e atitudes para o bom desempenho da função de Caixa Executivo e outra para julgar o quanto o ocupante deste cargo já dispunha para desempenhar a função.

Enviaram-se 898 questionários de avaliação de Caixa Executivo, sendo que 449 foram para os próprios e 449 para os Supervisores destes. Cada Supervisor respondeu uma média de 10 questionários de hetero-avaliação. Foram devolvidos um total de 669 (75\%) questionários, sendo 325 devidamente respondidos pelos Caixas Executivos e 344 pelos Supervisores dos Caixas Executivos.

\section{Procedimentos de análise de dados}

Para atingir o primeiro objetivo deste estudo, que foi o de desenvolver uma metodologia de avaliação de necessidades que também incluísse atitudes, antes se calcularam as necessidades de conhecimentos, habilidades e atitudes através das seguintes fórmulas propostas por Borges-Andrade e Lima (1983):

$$
\begin{aligned}
& \text { 1) } \mathrm{N}=\mathrm{i} \times(4-\mathrm{q}) \text { e 2) } \mathrm{N}=\mathrm{i} \times(4-\mathrm{d}) \\
& \text { Onde: } \\
& \begin{array}{l}
\mathrm{N}=\text { necessidade } \\
\mathrm{i}=\text { importância } \\
\mathrm{q}=\text { quantidade } \\
\mathrm{d}=\text { domínio }
\end{array}
\end{aligned}
$$

Para as necessidades de conhecimento utilizou-se a primeira fórmula e para as necessidades de habilidades e atitudes a segunda fórmula. O que difere a primeira da segunda é que para conhecimento nomeou-se a escala de "quantidade" de conhecimento, enquanto que para as habilidades e atitudes nomeou-se de "domínio". Com esta fórmula, definiu-se operacionalmente necessidades como sendo o produto da importância de conhecimentos, habilidades e atitudes, pela ausência de quantidade ou domínio desses conhecimentos, habilidades e atitudes. A mesma fórmula foi utilizada para estabelecer as necessidades avaliadas pelos Supervisores. Esta definição é consistente com os conceitos de necessidade de treinamento propostos por Morrisson (1977, citado por Nogueira, 1982) e por Borges-Andrade (1986), na medida em que a mencionada ausência equivale à noção de diferença ou discrepância entre desempenhos esperados e reais. Além disso, ela agrega a proposta de que esta discrepância precisa ser valorada (ou multiplicada) pela importância atribuída aos referidos desempenhos. Finalmen- te, ela supõe que estes desempenhos estejam baseados em conhecimentos, habilidades ou atitudes, tal como já tinha sido definido pelo primeiro destes dois autores e tem sido defendido pelos autores já citados de psicologia instrucional que desenvolveram taxonomias de objetivos instrucionais ou de capacidades subordinadas de aprendizagem. Os escores "N" resultantes podiam variar num intervalo de 0 a 16 .

Após estimar os escores das necessidades, realizou-se com eles a Análise dos Componentes Principais, com os dados dos Caixas Executivos e com os dos seus Supervisores. O critério escolhido para determinar o número de fatores no modelo foi o eigenvalue acima de 1 . Utilizou-se a rotação varimax, após se verificar a inexistência de correlações entre os fatores. Os valores ausentes foram substituídos pelas médias. Suprimiu-se, dos fatores, as variáveis com cargas menores que 0,35 . Para a análise de confiabilidade optou-se pelo Alpha de Cronbach. Verificou-se a correlação inter-item e o Alpha da escala se o item fosse retirado. O nível de confiabilidade mínimo utilizado para este estudo foi de 0,80 .

\section{Resultados}

Os resultados obtidos na análise foram um conjunto de oito fatores, no caso da auto-avaliação de necessidades dos Caixas Executivos. Após a análise de confiabilidade eliminou-se um item no fator 6 (cálculos) e um no fator 7 (controle e manutenção de cédulas) porque suas presenças diminuíam as confiabilidades dos fatores. Verificou-se que o conteúdo do fator 1 (atitudes do caixa executivo), é predominantemente de necessidades de atitudes para o desempenho da função de Caixa Executivo. O que predomina nos fatores 2 (preparação para atender clientes) e 8 (conhecimento técnico) são necessidades de conhecimentos. Nos fatores 3 (atendimento aos clientes), 4 (organização de documentos), 6 (cálculos) e 7 (controle e manutenção de cédulas), só há necessidades de habilidades. O conteúdo do fator 5 (rotinas da função de Caixa Executivo) é um agrupamento de necessidades de conhecimentos e habilidades relativas a rotinas do cargo.

Para a hetero-avaliação de necessidades de treinamento dos Caixas Executivos, os resultados da análise fatorial foram um conjunto de oito fatores, sendo que depois da análise de confiabilidade eliminou-se os dois últimos fatores por não levarem a indicadores confiáveis, ficando 6 fatores para a hetero-avaliação. No fator 2 (organização com as tarefas), eliminou-se um item após a análise de confiabilidade. No fator 3 (rotinas da função de Caixa Executivo), foram igualmente eliminados dois itens por não obterem escores confiáveis. Pelo mesmo motivo eliminou-se um item no fator 6 (cálculos).

Observou-se que no fator 1 (atendimento aos clientes) há um predomínio de necessidade de atitudes. No fator 2 
(organização das tarefas), há um agrupamento de necessidades de habilidades e atitudes. O conteúdo do fator 3 (rotinas da função de Caixa Executivo) é um agrupamento de habilidades e conhecimentos. No fator 4 (preparação para atender clientes), predominam necessidades de conhecimentos. No fator 5 (conhecimento técnico), só há necessidades de conhecimento e no fator 6 (cálculos), necessidades de habilidades.

Os resultados das análises anteriores, que são uma síntese daqueles descritos detalhadamente por Magalhães (1996), permitiram agregar as necessidades de treinamento dos Caixas Executivos nos fatores já citados. Estas médias foram estimadas calculando-se a média dos escores de necessidades das variáveis contidas em cada fator resultante da análise fatorial, após a eliminação dos itens não confiáveis. Com isso criou-se um escore global de necessidade para cada fator.

Fez-se a análise descritiva para auto e hetero-avaliações de necessidades de treinamento de Caixas Executivos. Os resultados da auto-avaliação indicaram escores médios de necessidades entre 0,89 e 5,26 (desvios-padrão entre 1,81 e $2,97)$. Nas hetero-avaliações, as médias de necessidades variaram entre 1.42 e 5.68 (desvios-padrão entre 2.07 e 2.96). Observou-se que as três primeiras e a última auto-avaliação de necessidades coincidiram exatamente com as hetero-avaliações. Até a décima primeira necessidade de auto-avaliação, verificou-se uma relação com a hetero-avaliação: as necessidades foram as mesmas, porém com ordenação li- geiramente diferente. As médias destas necessidades são geralmente maiores para a hetero-avaliação, mas há um mesmo nível de priorização delas em ambas avaliações. Todos esses resultados descritivos também estão disponíveis em Magalhães (1996).

O segundo objetivo deste estudo foi investigar a relação entre auto e hetero-avaliação, incluindo-se conhecimentos, habilidades e atitudes definidos para os Caixas Executivos. Para isto, utilizou-se a correlação de Pearson com teste de significância bicaudal. Para analisar as diferenças entre auto e hetero-avaliação, utilizou-se o teste $T$ de Student de amostras pareadas. $O$ índice de significância utilizado neste estudo, para a correlação de Pearson e para o Teste T, foi de $\mathrm{p}<0,01$.

Verificou-se, em todos os itens de conhecimento, correlações significativas (vide Tabela 1); existindo portanto relação entre auto e hetero-avaliações de necessidades de treinamento. Na Tabela 2, estão os mesmos resultados para habilidades. Verificou-se que os itens de número 3 (atender clientes), 4 (orientar clientes), 9 (controlar documentos relacionados com as atividades), 13 (efetuar rotinas de pagamentos) e 14 (efetuar rotinas de recebimentos) não tiveram correlações significativas ( $p>0,01$ ). Para estes itens não houve relação entre auto e hetero-avaliação. As correlações, tanto dos conhecimentos quanto das habilidades, não foram grandes. Para todos os itens de necessidades de atitudes a correlação também foi significativa, porém o " $\mathrm{r}$ " foi baixo (Tabela 3$)$.

Tabela 1.

Correlações entre auto-avaliação e avaliação pelo supervisor das necessidades de treinamento de conhecimentos para a função de Caixa Executivo

$\begin{array}{lr}\text { Conhecimentos } & \text { Correlações } \\ \text { Auto/Hetero }\end{array}$

\begin{tabular}{lll}
\hline 1. Conhecer as rotinas de pagamentos & $\mathrm{r}=.1985$ & $\mathrm{p}=.0001$ \\
2. Conhecer as rotinas de recebimentos & $\mathrm{r}=.2601$ & $\mathrm{p}=.0001$ \\
3. Ter conhecimento de grafoscopia & $\mathrm{r}=.2326$ & $\mathrm{p}=.0001$ \\
4. Conhecer as normas de segurança bancária & $\mathrm{r}=.2868$ & $\mathrm{p}=.0001$ \\
5. Ter conhecimento de datiloscopia & $\mathrm{r}=.3219$ & $\mathrm{p}=.0001$ \\
6. Conhecer as normas de fornecimento de talões de cheques & $\mathrm{r}=.3616$ & $\mathrm{p}=.0001$ \\
7. Saber normas de compensação de cheques e outros documentos & $\mathrm{r}=.3753$ & $\mathrm{p}=.0001$ \\
8. Conhecer de normas de arquivo & $\mathrm{r}=.2093$ & $\mathrm{p}=.0001$ \\
9. Conhecer as normas para expurgar contas encerradas & $\mathrm{r}=.2395$ & $\mathrm{p}=.0001$ \\
10. Ter conhecimento dos produtos da CEF & $\mathrm{r}=.2036$ & $\mathrm{p}=.0001$ \\
11. Conhecer todos tipos de aplicações financeiras existentes & $\mathrm{r}=.2762$ & $\mathrm{p}=.0001$ \\
\hline
\end{tabular}


Tabela 2.

Correlações entre auto-avaliação e avaliação pelo supervisor das necessidades de treinamento de habilidades para a função de Caixa Executivo

\begin{tabular}{lll}
\hline \multicolumn{1}{c}{ Habilidades } & \multicolumn{2}{c}{ Correlações } \\
& & \multicolumn{2}{c}{ Auto/Hetero } \\
\hline 1. Recompor cédulas dilaceradas & $\mathrm{r}=.1900$ & $\mathrm{p}=.001$ \\
2. Recolher cédulas dilaceradas & $\mathrm{r}=.2334$ & $\mathrm{p}=.0001$ \\
3. Atender clientes & $\mathrm{r}=.0997$ & $\mathrm{p}=.079$ \\
4. Orientar clientes & $\mathrm{r}=.1245$ & $\mathrm{p}=.028$ \\
5. Fornecer saldos e informações a respeito das contas dos clientes & $\mathrm{r}=.1851$ & $\mathrm{p}=.001$ \\
6. Controlar e guardar talões de cheque & $\mathrm{r}=.3655$ & $\mathrm{p}=0001$ \\
7. Efetuar cálculos & $\mathrm{r}=.2235$ & $\mathrm{p}=.0001$ \\
8. Conferir cálculos & $\mathrm{r}=.2270$ & $\mathrm{p}=.0001$ \\
9. Controlar documentos relacionados com as atividades & $\mathrm{r}=.1028$ & $\mathrm{p}=.070$ \\
10. Conferir documentos relacionados com as atividades & $\mathrm{r}=.2143$ & $\mathrm{p}=.0001$ \\
11. Guardar documentos relacionados com as atividades & $\mathrm{r}=.2006$ & $\mathrm{p}=.0001$ \\
12. Movimentar e controlar numerários, títulos e valores & $\mathrm{r}=.1666$ & $\mathrm{p}=.003$ \\
13. Efetuar rotinas de pagamentos. & $\mathrm{r}=.0942$ & $\mathrm{p}=.096$ \\
14. Efetuar rotinas de recebimentos & $\mathrm{r}=.1437$ & $\mathrm{p}=.011$ \\
15. Operar a máquina registradora & $\mathrm{r}=.1705$ & $\mathrm{p}=.002$ \\
\hline
\end{tabular}

Tabela 3

Correlações entre auto-avaliação e avaliação pelo supervisor das necessidades de treinamento de atitudes para a função de Caixa Executivo

$\begin{array}{ll}\text { Atitudes } & \text { Correlações } \\ \text { Auto/Hetero }\end{array}$

\begin{tabular}{lll}
\hline 1. Estar preparado(a) para dar informações adequadas & $\mathrm{r}=.2561$ & $\mathrm{p}=.0001$ \\
2. Demonstrar equilíbrio emocional & $\mathrm{r}=.1550$ & $\mathrm{p}=.006$ \\
3. Demonstrar interesse pelo trabalho & $\mathrm{r}=.1845$ & $\mathrm{p}=.001$ \\
4. Ser paciente ao tratar os clientes & $\mathrm{r}=.2318$ & $\mathrm{p}=.0001$ \\
5. Demonstrar agilidade no atendimento aos clientes & $\mathrm{r}=.3774$ & $\mathrm{p}=.0001$ \\
6. Ser organizado(a) no desenvolvimento das tarefas & $\mathrm{r}=.3354$ & $\mathrm{p}=.0001$ \\
7.Trabalhar com qualidade & $\mathrm{r}=.2505$ & $\mathrm{p}=.0001$ \\
8. Estar atento(a) ao que está executando & $\mathrm{r}=.2109$ & $\mathrm{p}=.0001$ \\
\hline
\end{tabular}


Quanto às diferenças entre médias de auto e hetero-avaliação para o conjunto de itens de conhecimento, observouse que não houve diferença significativa nos itens de números 1 (conhecer as rotinas de pagamentos), 4 (conhecer as normas de segurança bancária), 5 (ter conhecimento de datiloscopia) e 11 (conhecer todos os tipos de aplicações financeiras existentes). Nos demais, a diferença foi significativa, sendo que nos itens de número 2 (conhecer as rotinas de recebimentos), 6 (conhecer as normas de fornecimento de talões de cheques), 7 (saber normas de compensação de cheques e outros documentos), 8 (conhecer normas de arquivo), 9 (conhecer as normas para expurgar contas encerradas) e 10 (ter conhecimento dos produtos da CEF) a média maior foi para o Supervisor e, no item 3 (ter conhecimento de grafoscopia), para o Caixa Executivo.

Verificou-se que não houve diferença significativa para os itens relacionados com as habilidades de número 1 (recompor cédulas dilaceradas) e 12 (movimentar e controlar numerários, títulos e valores). Os outros itens tiveram diferenças significativas, com média maior para o Supervisor. Os itens de atitudes necessárias para o desempenho da função de Caixa Executivo que não apresentaram diferenças significativas foram os de números 1 (estar preparado/a para dar informações adequadas) e 2 (demonstrar equilíbrio emocional). Nos demais, outra vez os supervisores apareceram com médias maiores.

Utilizou-se a análise de regressão múltipla para verificar as variáveis preditoras dos escores globais de cada fator extraído da análise fatorial feita somente com as auto-avaliações dos Caixas Executivos. Observou-se que a variável tempo de trabalho na CEF apareceu para os fatores 2 (preparação para atender clientes), 3 (atendimento aos clientes) e 4 (organização de documentos): quanto menos tempo de trabalho na CEF, mais o respondente necessita de treinamento nestes fatores. Apareceu a variável gênero como preditora dos fatores 6 (cálculos) e 7 (controle) e manutenção de cédulas. Neste último, a variável tempo na função também apareceu. No que se refere à variável gênero, os Caixas Executivos do sexo feminino necessitam de mais treinamento nos conteúdos destes fatores. Quanto a tempo na função, quanto menor a experiência na mesma, mais o respondente necessita de treinamento nos conteúdos do fator 7 (controle e manutenção de cédulas).

As estatísticas relativas às análises inferenciais feitas, para verificar as diferenças entre avaliações e suas variáveis preditoras, estão integralmente disponíveis em Magalhães (1996).

\section{Discussão}

A estratégia de mensuração aqui utilizada, a partir da combinação de indicadores de julgamento de importância e quantidade ou domínio, tratou de manter uma estreita con- sistência com os conceitos de necessidades disponíveis na literatura anteriormente mencionada. O tratamento estatístico realizado, por sua parte, indicou consistência interna dos fatores e confiabilidade das referidas medidas.

Os resultados do presente estudo mostraram que a metodologia de avaliação de necessidades de treinamento, incluindo conhecimentos, habilidades e atitudes, parece ser adequada. Esta mesma metodologia, sem incluir a avaliação de atitudes, foi utilizada em pesquisas anteriores e demonstrou ser adequada para estudar necessidades de treinamento. Como foi antes mencionado, há uma evidente inconsistência na literatura científica sobre treinamento, que adota a maior parte do referencial da psicologia instrucional, mas que não inclui "atitudes" nos métodos disponíveis de avaliação de necessidades. Demonstrou-se aqui que isto é viável e que os resultados podem permanecer consistentes e confiáveis.

Foram extraídos fatores confiáveis para auto e heteroavaliação. Verificou-se, entretanto, a existência de algumas diferenças entre as análises fatoriais, realizadas para os dois tipos de avaliações, a começar pelo número de fatores extraídos para auto-avaliação e para hetero-avaliação. Outra diferença está na maneira como algumas necessidades se agruparam nos fatores. As respostas dos Caixas Executivos agruparam-se de acordo com o modelo utilizado para a construção do questionário, separando as categorias de conhecimentos, habilidades e atitudes. Dos 8 fatores extraídos, apenas nos fatores 2 (preparação para atender clientes) e 5 (rotinas da função de Caixa Executivo) as necessidades misturam itens destas categorias. Percebeu-se que as necessidades agrupadas nestes dois fatores estão relacionadas entre si, formando um conjunto de atividades inerentes à função de Caixa Executivo, que são: preparação para atender clientes e rotinas da função de Caixa Executivo.

As respostas dos Supervisores agruparam necessidades de uma maneira que se formou um conjunto de atividades que se assemelham em suas descrições e que juntas têm uma finalidade bem própria da CEF: atender clientes, organizar tarefas e cuidar de rotinas, nesta ordem. Na heteroavaliação, ao contrário da auto, em apenas 2 fatores (5 [conhecimento técnico] e 6 [cálculos]), as necessidades préclassificadas em termos de atitudes, habilidades e conhecimentos não se misturaram. Para os Supervisores, portanto, os processos psicológicos envolvidos nestas três categorias estão inter-relacionados e é mais fácil separá-los em função das finalidades organizacionais citadas. Os Caixas Executivos, por outro lado, conseguem fazer suas auto-avaliações dentro dos limites destas categorias, talvez por se tratarem de processos psicológicos aos quais têm acesso subjetivo. $\mathrm{Na}$ ausência de acesso direto a esses processos, as heteroavaliações dos Supervisores acabam sendo agregadas a partir 
de parâmetros externos que eles conhecem bem: os das finalidades organizacionais.

Os fatores 2 (preparação para atender clientes), 3 (atendimento aos clientes), 5 (rotinas da função de Caixa Executivo) e 6 (cálculos) da auto-avaliação assemelham-se, respectivamente, aos fatores 4 (preparação para atender clientes), 1 (atendimento aos clientes), 3 (rotinas da função de Caixa Executivo) e 6 (cálculos) da hetero-avaliação. Estes fatores são: atendimento aos clientes, rotinas da função de Caixa Executivo, preparação para atender clientes e cálculos. Verificou-se, portanto, uma relação entre auto e heteroavaliação no que se refere aos quatro fatores citados, embora as percepções e os julgamentos possam também ter suas diferenças, como ficou demonstrado nos dois parágrafos anteriores.

Este estudo também teve como objetivos estudar as correlações e as diferenças entre auto e hetero-avaliação, o que, conforme inicialmente afirmado aqui, não parece ter sido antes investigado no Brasil. Para o estudo das correlações entre a auto-avaliação e a avaliação feita pelo supervisor, buscando avaliar conhecimentos, habilidades e atitudes, os resultados das análises dos dados demonstraram que em todos os itens de conhecimentos e atitudes foram encontradas correlações significativas. Existe portanto uma relação entre auto e hetero-avaliação de necessidades de treinamento, embora as correlações não sejam elevadas. Quanto aos itens de habilidades, 5 dos 15 itens não tiveram correlações significativas. Como são itens relacionados com atividades rotineiras dos Caixas Executivos, uma das razões pode ser que os respondentes não se avaliariam como necessitando destas habilidades por serem habilidades específicas da função de Caixa Executivo e que no mínimo todo ocupante deveria saber, principalmente porque os ocupantes têm mais de 3 anos na função. Outra razão pode ser que, à época em que os questionários foram respondidos, a CEF estava passando por uma restruturação interna, não se tendo conhecimento das mudanças que iriam ocorrer, havendo portanto um receio em apontar as reais necessidades mais diretamente relacionadas à função, por temor a conseqüências relativas à administração do pessoal. De qualquer maneira, foram confirmados os estudos internacionais aqui revisados, que encontraram correlações entre auto e hetero-avaliações de necessidades.

Quanto às diferenças entre a auto-avaliação e a avaliação feita pelo Supervisor, nos itens de conhecimento, quatro não tiveram diferenças significativas, enquanto que para os itens de habilidades e atitudes dois itens não tiveram diferenças significativas. Observou-se que os resultados mostraram com clareza que os Caixas Executivos e os Supervisores diferiram em suas avaliações. Alguns relatos de pesquisas explicam que diferenças entre auto-avaliação e hetero-avaliação podem ser esperadas, porque cada avaliador tem uma perspectiva única e focaliza diferentes facetas do desempenho do trabalho. Os Supervisores, quando diferem dos subordinados, tendem a apontar maiores necessidades de treinamento, o que igualmente confirma aqueles resultados das pesquisas realizadas no exterior, sintetizados no início do presente texto.

Encontrou-se relações significativas entre necessidades de treinamento de conhecimentos, habilidades e atitudes e algumas variáveis preditoras. A variável tempo de trabalho na CEF emergiu como a melhor preditora de necessidades de treinamento de habilidades. Verificou-se também este fato através de entrevista com o Instrutor dos Caixas, que afirmou que quanto mais tempo de organização, menos os Caixas acreditam necessitar de treinamentos relativos às habilidades específicas para a sua função. Observou-se que estas atividades, na análise descritiva, não foram analisadas como necessárias para um programa de treinamento. Para compreender a força da variável tempo de trabalho, no contexto da CEF, deve ser lembrado que esta organização segue há muito tempo uma política de selecionar seu pessoal para cargos de nível inicial e depois internamente promover sua carreira num processo que até recentemente durava até a aposentadoria.

Para necessidades de treinamento relativas a conhecimentos, apareceu a variável preditora tempo de trabalho na CEF nos itens relativos ao conhecimento das rotinas de recebimentos e normas de segurança bancária. Quando se tratam de atividades rotineiras da função de Caixa Executivo, quem teve emprego anterior à CEF necessita de menos treinamento nestas atividades. Tempo na função é outra variável preditora que apareceu nas necessidades de treinamento relativas a conhecimento. Percebeu-se que a experiência anterior ajuda no desenvolvimento das atividades ligadas a esses conhecimentos.

Nas necessidades de treinamento relativas às habilidades, quando se trata de cálculos, as pessoas do sexo feminino necessitam de mais treinamento. Quando a habilidade está ligada a controle e manutenção das cédulas, quem tem menos tempo na função necessita de treinamento nesta área.

No que se refere a atitudes, apareceram as variáveis sexo, tempo na função, escolaridade e emprego anterior à CEF, como preditoras. Quando se trata de organização no desenvolvimento das tarefas, quem não teve emprego anterior tem maior necessidade de treinamento nesta atitude. As pessoas com nível escolar mais elevado tem mais necessidade de treinamento no que se refere a paciência ao tratar os clientes e atenção no que estão executando. As pessoas do sexo feminino necessitam de treinamento no que concerne a agilidade no atendimento aos clientes e de estarem preparadas para dar informações adequadas. 
Uma das conclusões mais importantes a respeito destes resultados é que experiências anteriores e tempo de trabalho contribuíram fortemente para o desempenho satisfatório (ou redução das necessidades de treinamento) das atividades inerentes à função de Caixa Executivo. $\mathrm{O}$ fato de terem um certo tempo de trabalho pode fazer com que os ocupantes da referida função se acomodem. Como são atividades rotineiras, pode existir o receio de se afirmar o desconhecimento delas. Numa organização como a EMBRAPA, no entanto, outros fatores preditores são mais importantes, como natureza do papel organizacional e treinamentos formais anteriores, de acordo com os resultados de pesquisa anteriormente citada aqui (Borges-Andrade et al, 1989), feita entre gerentes. Resultado similar foi encontrado entre empresários por Ford e Noe (1987), cujas variáveis preditoras mencionadas no início do presente texto parecem estar relacionadas com a natureza do papel.

Outra conclusão importante é que existem correlações entre auto e hetero-avaliação de necessidades de treinamento. Mas as avaliações podem diferir quanto à intensidade, dependendo de quem as julga. Considerando que essas correlações positivas e essas diferenças significativas foram verificadas numa mesma base de dados, isso esclarece um pouco a aparente inconsistência entre resultados de distintas pesquisas, aqui relatadas. É muito provável que isto tenha ocorrido pelo fato de diferentes pesquisadores terem utilizados somente uma das duas análises estatísticas: de correlação ou de diferenças entre médias.

O presente estudo tem algumas limitações importantes a serem consideradas. A primeira é que foi analisada somente uma organização e a segunda é que o estudo só pode ser desenvolvido com um único cargo. A terceira é que a avaliação de necessidades foi um esforço concentrado num dos componentes do quadro de referências de McGehee e Thayer (1961) e de Goldstein (1991): análise de tarefas. Esforços de pesquisa precisam ser igualmente realizados para os componentes de análise organizacional e pessoal, visando a avaliação de necessidades de treinamento. A integração teórica e metodológica desses componentes, provavelmente através de uma abordagem multinível, é outro desafio apontado por Kozlowski et al. (2000), para o qual pouco esforço foi direcionado.

Com base nos resultados deste estudo, futuras pesquisas devem continuar a explorar simultaneamente o grau de acordo e diferenças entre auto e hetero-avaliações, incluindo mais organizações e diferentes níveis e tipos de cargos. Para a Caixa Econômica Federal de Goiás, sugere-se que esta metodologia para analisar necessidades de treinamento seja realizada para todos os cargos da instituição, comparando as avaliações e discutindo os resultados com os avaliandos e avaliadores, com a finalidade de entrarem em um consenso e melhorarem os seus programas de treinamento. Finalmente, considerando-se as profundas mudanças ocorridas nas organizações bancárias, seria muito oportuno realizar análises de tarefas focadas em competências esperadas no futuro, como foi feito no Banco do Brasil, por Brandão (1999).

\section{Agradecimentos}

Os autores agradecem ao CNPq pelo apoio, na forma de Bolsa de Mestrado para a primeira autora e de Produtividade em Pesquisa para o segundo, e à CEF pelo suporte institucional para a realização da pesquisa.

\section{Referências}

Borges-Andrade, J. E. (1986). Por uma competência política e técnica em treinamento. Psicologia: Ciência e Profissão, 6(2), 9-17.

Borges-Andrade, J. E., \& Abbad-Oc, G (1996). Treinamento no Brasil: reflexões sobre suas pesquisas. Revista de Administração, 31(2), 112125.

Borges-Andrade, J. E., \& Lima, S. M. V. (1983). Avaliação de necessidades de treinamento: um método de análise de papel ocupacional. Tecnologia Educacional, 12(54), 5-14.

Borges-Andrade, J. E., Lima, S. M. V., Soares, C. R. V., \& Paula, S. M. A. (1989). Treinamento de gerentes de pesquisa: necessidades e impacto. Cadernos de Difusão de Tecnologia, 6(1), 117-139.

Byhan, W. C. (1977). Changing supervisory and managerial behavior: II. Training and Development Journal, 31(5), 10-16.

Brandão, H. P. (1999). Gestão baseada nas competências: um estudo sobre a identificação de competências profissionais na indústria bancária. Dissertação de mestrado não-publicada, Universidade de Brasília, Brasília, DF.

Carvalho, L. C. F. (1999). T\&D Estratégicos. In G. G. Boog (Org.), Manual de treinamento e desenvolvimento (pp. 125-144). São Paulo: Makron.

Chiavenato, I. (1992). Recursos humanos (ed. compacta). São Paulo: Atlas.

Ford, J. K., \& Noe, R. A. (1987). Self-assessed training needs: effects of attitudes toward training, managerial, level, and function. Personnel Psychology, 40(1), 39-53.

Gagné, R. M., \& Briggs, L. J. (1974). Principles of instructional design. New York: Holt, Rimehart and Winston.

Goldstein, I. L. (1991). Training in work organizations. In M. D. Dunnette \& L. M. Hough (Orgs.), Handbook of industrial and organizational psychology (pp. 507-619). Palo Alto: Consulting Psych.

Kozlowski, S. W. J., Brown, K. G., Weissbein, D. A., Cannon-Bowers, J. A., \& Salas, E. (2000). A Multilevel approach to training effectiveness: enhancing horizontal and vertical transfer. In K. J. Klein \& S. W. J. Koslowski (Orgs.), Multilevel theory, research, and methods in organizations (pp 157-210). San Francisco: Jossey-Bass.

Magalhães, M. L. (1996). Auto e hetero-avaliação no diagnóstico de necessidades de treinamento. Dissertação de mestrado não-publicada, Universidade de Brasília, Brasília, DF.

Mcenery, J., \& Mcenery, J. M. (1987). Self-rating in management training needs assessment: A neglected opportunity? Journal of Occupational Psychology, 60, 49-60.

McGehee, W., \& Tahyer, P. W. (1961). Training in business and industry. New York: Wiley.

Menezes, L. A., Rivera, R. C. P., \& Borges-Andrade, J. E. (1988). Necessidades de treinamento de agricultores e de suas mulheres num proje- 
to integrado de colonização. Cadernos de Difusão de Tecnologia, 5(1/ 3), 109-118.

Morano, R. (1973) Determining organizational training needs. Personnel Psychology, 26(4), 479-487.

Nogueira, P. R. (1980). Identificação das necessidades de treinamento: um estudo sobre os efeitos da posição hierárquica. Dissertação de mestrado não-publicada, Universidade de Brasília, Brasília, DF.

Nogueira, P. R. (1982). Necessidade de treinamento: construção e validação de um instrumento. Arquivos Brasileiros de Psicologia, 34(4), 46-65.

Ostroff, C., \& Ford, J. K. (1989). Assessing training needs: critical levels of analysis. In I. Goldstein (Org.), Training and development in organizations (pp. 25-62). San-Francisco: Jossey Bass.

Paula, S. M. A. (1992). Variáveis preditoras de impacto de treinamento no trabalho: análise da percepção dos treinados de duas organizações.
Dissertação de mestrado não-publicada, Universidade de Brasília, Brasília, DF.

Rothwell, W. J., \& Kazanas, H. C. (1992). Mastering the instructional design process. San Francisco: Jossey-Bass.

Schneider, B. (1999). Is the sky really falling? A View of the future. In A. I. Kraut \& A. K. Korman (Orgs.), Evolving Practices in Human Resource Management (pp. 328-357). San Francisco: Jossey-Bass.

Stanley, C., \& Shockley-Zalabak, P. (1986). Communication proficiency and future training needs of the female professional: self-assessment vs. supervisors' evaluations. Human Relations, 39(10), 891-902.

Yoder, D. (1956). Administração de pessoal e relações industriais. São Paulo: Mestre Jou.

Wexley, K. N. (1984). Personnel training. Annual Review of Psychology, 35, 519-551.

Waite, W. (1952). Personnel Administration. New York: Ronald..

Nota

Os dados referentes à empresa foram fornecidos por funcionários, durante entrevistas preliminares e de acompanhamento.

Mônica Lemes Magalhães, mestre em Psicologia Social e do Trabalho pela Universidade de Brasília, trabalha na área de recursos humanos há 13 anos e é professora no Departamento de Psicologia da Faculdade Garcia Silveira.

Endereço para correspondência: QRSW 2 - Bloco A-14 - Apt ${ }^{\circ} 305$ - Sudoeste. Brasília, DF. E-mail: mlmagal@uol.com.br. Fone: (61) 344-5078. Fax: (61) 212-8580.

Jairo Eduardo Borges-Andrade, doutor em Sistemas Instrucionais pela Florida State University (USA), durante 14 anos, pesquisador da EMBRAPA nas áreas de recursos humanos e de administração estratégica, é Professor Titular no Departamento de Psicologia Social e do Trabalho do Instituto de Psicologia da Universidade de Brasília.

Endereço para correspondência: SQN 106 - I - 605 - 70742-090 Brasília, DF. E-mail: jeborges@linkexpress.com.br. Fone: (61) 307-2625 (r. 221). Fax: (61) 273-0203. 


\section{APÊNDICE A}

\section{QUESTIONÁRIO DE AVALIAÇÃO DE NECESSIDADES DE TREINAMENTO DE CAIXA EXECUTIVO - AVALIAÇÃO PELO SUPERVISOR}

Este instrumento foi elaborado com o objetivo de conhecer as necessidades de treinamento para o bom desempenho do caixa executivo em suas atribuições. As informações serão utilizadas pelo escritório de negócios, para melhorar o treinamento dos caixas. Elas serão mantidas em sigilo e só serão analisadas de forma agregada.

Nome do Caixa Executivo cujas necessidades estão sendo avaliadas:

Nome do(a) Avaliador(a):

Agência:

Idade: Sexo:

Estado Civil: Escolaridade:

Experiências Anteriores (cargos ocupados em outras empresas):

Treinamentos que participou antes de entrar na CEF:

Tempo de Serviço na CEF:

Tempo de Serviço no cargo atual:

Treinamentos que você participou dentro da CEF:

Funções que você desenvolveu dentro da CEF:

Tempo de permanência em cada função citada acima:

Treinamentos oferecidos pela CEF que você tem interesse em participar: 
Abaixo há uma lista de conhecimentos necessários para o bom desempenho das atribuições de um caixa executivo. Você deverá avaliar cada um destes conhecimentos usando duas escalas de julgamento.

Na primeira escala você deverá julgar a importância destes conhecimentos, escrevendo o número de sua resposta na coluna $\mathbf{I}$, ao lado de cada conhecimento descrito. Para isso utilize a seguinte escala:

0 - sem importância

1 - pouco importante

2 - importância média

3 - importante

4 - muito importante

Na coluna $\mathbf{Q}$ você vai julgar a quantidade de determinado conhecimento que o caixa executivo acima citado já dispõe para desempenhar a função. Use a escala abaixo:

0 - não tem qualquer conhecimento

1 - tem aproximadamente $25 \%$ do conhecimento necessário

2 - tem aproximadamente $50 \%$ do conhecimento necessário

3 - tem aproximadamente $75 \%$ do conhecimento necessário

4 - tem todo o conhecimento necessário

Ao final, verifique se não deixou nenhum item sem responder.

1. Conhecer as rotinas de pagamentos.

( ) ( )

2. Conhecer as rotinas de recebimentos.

( ) ( )

3. Ter conhecimento de grafoscopia.

$($ ) $($ )

4. Conhecer as normas de segurança bancária.

( ) ( )

5. Ter conhecimento de datiloscopia.

$($ ) $($ )

6. Conhecer as normas de fornecimento de talões de cheques.

( ) ( )

7. Saber normas de compensação de cheques e outros documentos. $\quad$ ( ) ( )

8. Conhecer normas de arquivo.

( ) ( )

9. Conhecer as normas para expurgar contas encerradas. $\quad($ ) ( )

10. Ter conhecimento dos produtos da CEF. $\quad($ ) ( )

11. Conhecer todos os tipos de aplicações financeiras existentes.

$($ ) $($ )

Escreva outros conhecimentos para os quais você julga que ele(a) possui necessidades de treinamento: 
Abaixo está uma lista de habilidades necessárias para o bom desempenho das atribuições de um caixa executivo. Você deverá avaliar cada uma destas habilidades usando duas escalas de julgamento.

Primeiro você deverá julgar a importância destas habilidades para o desempenho da função. Para isso utilize o mesmo procedimento anterior.

Na coluna D você irá julgar o quanto o caixa executivo acima citado já domina esta habilidade. Use a escala abaixo:

\section{0 - não domina}

\section{1 - possui pouco domínio}

2 - possui domínio médio

\section{3 - domina quase totalmente}

4 - domina totalmente

01. Recompor cédulas dilaceradas.

02. Recolher cédulas dilaceradas.

03. Atender clientes.

04. Orientar clientes.

05. Fornecer saldos e informações a respeito das contas dos clientes.

06. Controlar e guardar talões de cheque.

07. Efetuar cálculos.

08. Conferir cálculos.

09. Controlar documentos relacionados com as atividades.

10. Conferir documentos relacionados com as atividades.

11. Guardar documentos relacionados com as atividades.

12. Movimentar e controlar numerários, títulos e valores.

13. Efetuar rotinas de pagamentos.

14. Efetuar rotinas de recebimentos.

15. Operar a máquina registradora.

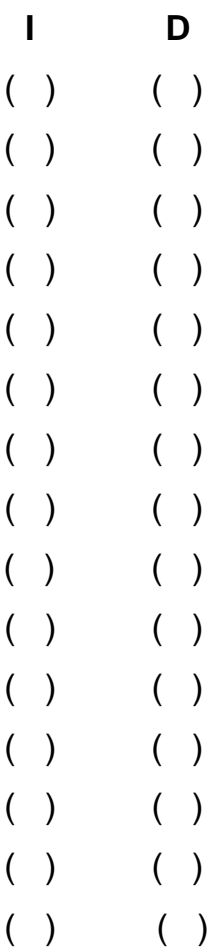

Escreva outras habilidades para as quais você julga que ele(a) possui necessidades de treinamento:

Abaixo está uma lista de atitudes necessárias para o bom desempenho das atribuições de um caixa executivo. Você deverá avaliar cada uma delas usando duas escalas de julgamento. 
Primeiro você deverá julgar a importância destas atitudes para o bom desempenho da função. Para isso utilize o mesmo procedimento anterior.

Na coluna D você vai julgar o quanto o caixa executivo acima citado já possui desenvolvida esta atitude. Use a escala abaixo:

\section{0 - não tem muito desenvolvida}

\section{1 - tem pouco desenvolvida}

2 - tem medianamente desenvolvida

\section{3 - tem muito desenvolvida}

\section{4 - tem desenvolvida totalmente}

1. Estar preparado(a) para dar informações adequadas.

I D

2. Demonstrar equilíbrio emocional.

3. Demonstrar interesse pelo trabalho.

4. Ser paciente ao tratar os clientes.

5. Demonstrar agilidade no atendimento aos clientes.

6. Ser organizado(a) no desenvolvimento das tarefas.

7. Trabalhar com qualidade.

8. Estar atento(a) ao que está executando.

( ) ( )

( ) ( )

( ) ( )

$($ ) ( )

$($ ) $($ )

$($ ( ) $($ )

( ) ( )

( ) ( )

Escreva outras atitudes para as quais você julga que ele(a) possui necessidade de treinamento: 